\title{
Dark Stars: Dark matter in the first stars leads to a new phase of stellar evolution
}

\author{
Katherine Freese $^{1}$, Douglas Spolyar ${ }^{2}$, Anthony Aguirre ${ }^{2}$, Peter \\ Bodenheimer $^{3}$, Paolo Gondolo ${ }^{4}$, J. A. Sellwood ${ }^{5}$, and Naoki Yoshida ${ }^{6}$ \\ ${ }^{1}$ Michigan Center for Theoretical Physics, University of Michigan, Ann Arbor, MI 48109, USA \\ email: ktfreese@umich.edu \\ ${ }^{2}$ Dept. of Physics, University of California, Santa Cruz, CA 95064, USA \\ email: dspolyar@physics.ucsc.edu, aguirre@scipp.ucsc.edu \\ ${ }^{3}$ Dept. of Astronomy, University of California, Santa Cruz, CA 95064, USA \\ email: peter@ucolick.org \\ ${ }^{4}$ Physics Dept., University of Utah, Salt Lake City, UT 84112, USA \\ email: paolo@physics.utah.edu \\ ${ }^{5}$ Dept. of Physics and Astronomy, Rutgers Univ., Piscataway, NJ 08854, USA \\ email: sellwood@physics.rutgers.edu \\ ${ }^{6}$ Inst. for the Physics and Math. of the Universe, Univ. of Tokyo, Kashiwa, Chiba, Japan \\ email: nyoshida@a.phys.nagoya-u-ac.jp
}

\begin{abstract}
The first phase of stellar evolution in the history of the universe may be Dark Stars, powered by dark matter heating rather than by fusion. Weakly interacting massive particles, which are their own antiparticles, can annihilate and provide an important heat source for the first stars in the the universe. This talk presents the story of these Dark Stars. We make predictions that the first stars are very massive $\left(\sim 800 M_{\odot}\right)$, cool $(6000 \mathrm{~K})$, bright $\left(\sim 10^{6} L_{\odot}\right)$, longlived ( $\sim 10^{6}$ years), and probable precursors to (otherwise unexplained) supermassive black holes. Later, once the initial DM fuel runs out and fusion sets in, DM annihilation can predominate again if the scattering cross section is strong enough, so that a Dark Star is born again.
\end{abstract}

Keywords. cosmology: dark matter, stars: evolution, stars: fundamental parameters

\section{Introduction}

In October 2006, as guests of the Galileo Galilei Institute in Florence, two of us began a new line of research: the effect of Dark Matter particles on the very first stars to form in the universe. We found a new phase of stellar evolution: the first stars to form in the universe may be "Dark Stars:" dark matter powered rather than fusion powered. We first reported on this work in a paper (Spolyar, Freese, \& Gondolo 2008) submitted to the arxiv in April 2007 (hereafter Paper I). When we presented this work at The First Stars conference in Santa Fe soon after (Freese, Gondolo \& Spolyar 2008), many questions were raised, which we have addressed in the subsequent year. In this talk I review the basic ideas as well as report on the followup work we performed over the past year.

The Dark Matter particles considered here are Weakly Interacting Massive Particles (WIMPs) (such as the Lightest Supersymmetric Particle), which are one of the major motivations for building the Large Hadron Collider at CERN that will begin taking data very soon. These particles are their own antiparticles; they annihilate among themselves in the early universe, leaving the correct relic density today to explain the dark matter in the universe. These particles will similarly annihilate wherever the DM density is high. The first stars are particularly good sites for annihilation because they form at high 


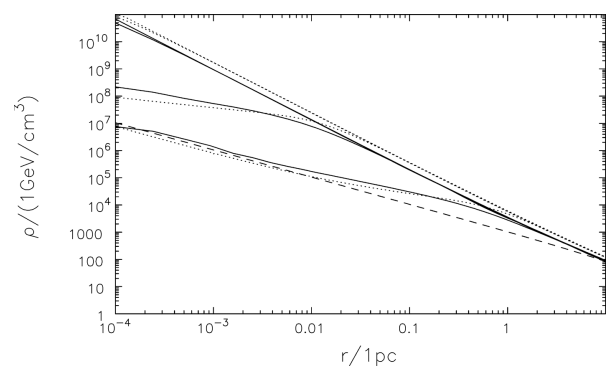

Figure 1. Adiabatically contracted DM profiles in the first protostars for an initial NFW profile (dashed line) using (a) the Blumenthal method (dotted lines) and (b) an exact calculation using Young's method (solid lines), for $M_{\mathrm{vir}}=5 \times 10^{7} M_{\odot}, c=2$, and $z=19$. The four sets of curves correspond to a baryonic core density of $10^{4}, 10^{8}, 10^{13}$, and $10^{16} \mathrm{~cm}^{-3}$. The two different approaches to obtaining the DM densities find values that differ by less than a factor of two.

redshifts (density scales as $(1+z)^{3}$ ) and in the high density centers of DM haloes. The first stars form at redshifts $z \sim 10-50$ in dark matter $(\mathrm{DM})$ haloes of $10^{6} M_{\odot}$ (for reviews see e.g. Ripamonti \& Abel 2005, Barkana \& Loeb 2001, Bromm \& Larson 2003; see also Yoshida et al. 2006). One star is thought to form inside one such DM halo.

As our canonical values, we will use the standard annihilation cross section, $\langle\sigma v\rangle=$ $3 \times 10^{-26} \mathrm{~cm}^{3} / \mathrm{sec}$, and $m_{\chi}=100 \mathrm{GeV}$ for the particle mass; but we will also consider a broader range of masses and cross-sections. Paper I found that DM annihilation provides a powerful heat source in the first stars, a source so intense that its heating overwhelms all cooling mechanisms; subsequent work has found that the heating dominates over fusion as well once it becomes important at later stages (see below). Paper I (Spolyar, Freese, \& Gondolo 2008) suggested that the very first stellar objects might be "Dark Stars," a new phase of stellar evolution in which the DM - while only a negligible fraction of the star's mass - provides the power source for the star through DM annihilation.

\section{Three Criteria}

Paper I (Spolyar, Freese, \& Gondolo 2008) outlined the three key ingredients for Dark Stars: 1) high dark matter densities, 2) the annihilation products get stuck inside the star, and 3) DM heating wins over other cooling or heating mechanisms. These same ingredients are required throughout the evolution of the dark stars, whether during the protostellar phase or during the main sequence phase.

First criterion: High Dark Matter density inside the star. To find the DM density profile, we start with an overdense region of $\sim 10^{6} M_{\odot}$ with an $\mathrm{NFW}$ (Navarro, Frenk \& White 1996) profile for both DM and gas, where the gas contribution is $15 \%$ of that of the DM. Originally we used adiabatic contraction $(M(r) r=$ constant) (Blumenthal et al. 1985) and matched onto the baryon density profiles given by Abel, Bryan \& Norman (2002) and Gao et al. (2007) to obtain DM profiles. This method is overly simplified: it considers only circular orbits of the DM particles. Our original DM profile matched that obtained numerically in Abel, Bryan, \& Norman (2002) with $\rho_{\chi} \propto r^{-1.9}$, for both their earliest and latest profiles; see also Natarajan, Tan, \& O'Shea (2008) for a recent discussion. Subsequent to our original work, we have done an exact calculation (which includes radial orbits, Freese, Gondolo, Sellwood \& Spolyar 2008) and found that our original results were remarkably accurate, to within a factor of two. Our resultant DM profiles are shown in Fig. 1. At later stages, we also consider possible further enhancements due to capture of DM into the star (discussed below). 


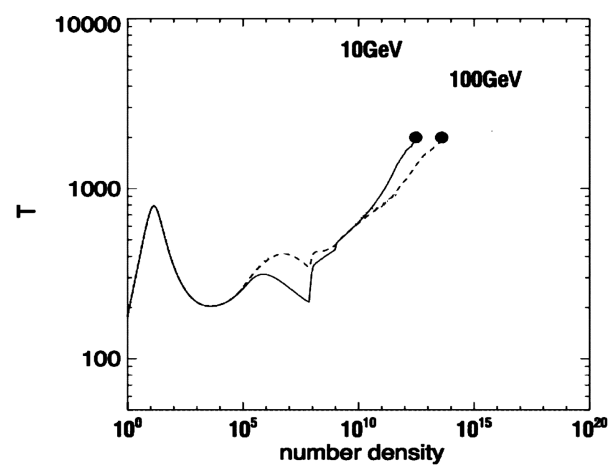

Figure 2. Temperature (in degrees K) as a function of hydrogen density (in $\mathrm{cm}^{-3}$ ) for the first protostars, with DM annihilation included, for two different DM particle masses (10 GeV and $100 \mathrm{GeV}$ ). Moving to the right in the figure corresponds to moving forward in time. Once the "dots" are reached, DM annihilation wins over H2 cooling, and a Dark Star is created.

Second Criterion: Dark Matter Annihilation Products get stuck inside the star. WIMP annihilation produces energy at a rate per unit volume $Q_{\text {ann }}=\langle\sigma v\rangle \rho_{\chi}^{2} / m_{\chi}$ $\simeq 1.2 \times 10^{-29} \mathrm{erg} / \mathrm{cm}^{3} / \mathrm{s}\left(\langle\sigma v\rangle /\left(3 \times 10^{-26} \mathrm{~cm}^{3} / \mathrm{s}\right)\right)\left(n / \mathrm{cm}^{-3}\right)^{1.6}\left(m_{\chi} /(100 \mathrm{GeV})\right)^{-1}$. In the early stages of Pop III star formation, when the gas density is low, most of this energy is radiated away (Ripamonti, Mapelli \& Ferrara 2006). However, as the gas collapses and its density increases, a substantial fraction $f_{Q}$ of the annihilation energy is deposited into the gas, heating it up at a rate $f_{Q} Q_{\text {ann }}$ per unit volume. While neutrinos escape from the cloud without depositing an appreciable amount of energy, electrons and photons can transmit energy to the core. We have computed estimates of this fraction $f_{Q}$ as the core becomes more dense. Once $n \sim 10^{11} \mathrm{~cm}^{-3}$ (for $100 \mathrm{GeV}$ WIMPs), $\mathrm{e}^{-}$and photons are trapped and we can take $f_{Q} \sim 2 / 3$.

Third Criterion: DM Heating is the dominant heating/cooling mechanism in the star. We find that, for WIMP mass $m_{\chi}=100 \mathrm{GeV}(1 \mathrm{GeV})$, a crucial transition takes place when the gas density reaches $n>10^{13} \mathrm{~cm}^{-3}\left(n>10^{9} \mathrm{~cm}^{-3}\right)$. Above this density, DM heating dominates over all relevant cooling mechanisms, the most important being $\mathrm{H}_{2}$ cooling (Hollenbach \& McKee 1979).

Figure 2 shows evolutionary tracks of the protostar in the temperature-density phase plane with DM heating included (Yoshida et al. 2008), for two DM particle masses $(10 \mathrm{GeV}$ and $100 \mathrm{GeV}$ ). Moving to the right on this plot is equivalent to moving forward in time. Once the black dots are reached, DM heating dominates over cooling inside the star, and the Dark Star phase begins. The protostellar core is prevented from cooling and collapsing further. The size of the core at this point is $\sim 17$ A.U. and its mass is $\sim 0.6 M_{\odot}$ for $100 \mathrm{GeV}$ mass WIMPs. A new type of object is created, a Dark Star supported by DM annihilation rather than fusion.

\section{Building up the Mass}

Recently, we have found the stellar structure of the dark stars (hereafter DS) (Freese, Bodenheimer, Spolyar, \& Gondolo 2008). Though they form with the properties just mentioned, they continue to accrete mass from the surrounding medium. In our paper we build up the DS mass as it grows from $\sim 1 M_{\odot}$ to $\sim 1000 M_{\odot}$. As the mass increases, the DS contracts and the DM density increases until the DM heating matches its radiated luminosity. We find polytropic solutions for dark stars in hydrostatic and thermal equilibrium. We start with a few $M_{\odot}$ DS and find an equilibrium solution. Then we build 


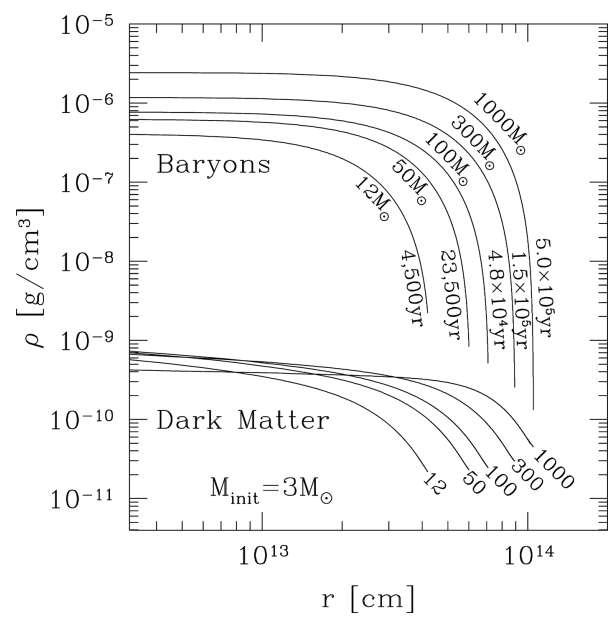

Figure 3. Evolution of a dark star $(\mathrm{n}=1.5)$ as mass is accreted onto the initial protostellar core of $3 \mathrm{M}_{\odot}$. The set of upper (lower) curves correspond to the baryonic (DM) density profile at different masses and times. Note that DM constitutes $<10^{-3}$ of the mass of the DS.

up the DS by accreting $1 M_{\odot}$ at a time with an accretion rate of $2 \times 10^{-3} M_{\odot} / \mathrm{yr}$, always finding equilibrium solutions. We find that initially the DS are in convective equilibrium; from $(100-400) M_{\odot}$ there is a transition to radiative; and heavier DS are radiative. As the DS grows, it pulls in more DM, which then annihilates. We continue this process until the DM fuel runs out at $M_{D S} \sim 800 M_{\odot}$ (for $100 \mathrm{GeV}$ WIMPs). Figure 3 shows the stellar structure. One can see "the power of darkness:" although the DM constitutes a tiny fraction $\left(<10^{-3}\right)$ of the mass of the DS, it can power the star. The reason is that WIMP annihilation is a very efficient power source: $2 / 3$ of the initial energy of the WIMPs is converted into useful energy for the star, whereas only $1 \%$ of baryonic rest mass energy is useful to a star via fusion.

\section{Results and Predictions}

Our final result (Freese, Bodenheimer, Spolyar, \& Gondolo 2008), is very large first stars! E.g., for $100 \mathrm{GeV}$ WIMPs, the first stars have $M_{D S}=800 M_{\odot}$. Once the DM fuel runs out inside the DS, the star contracts until it reaches $10^{8} \mathrm{~K}$ and fusion sets in. A possible end result of stellar evolution will be large black holes. The Pair Instability SN (Heger \& Woosley 2002) that would be produced from 140-260 $M_{\odot}$ stars (and whose chemical imprint is not seen) would not be as abundant. Indeed this process may help to explain the supermassive black holes that have been found at high redshift $\left(10^{9} M_{\odot}\right.$ $\mathrm{BH}$ at $\mathrm{z}=6$ ) and are, as yet, unexplained (Li et al. 2006; Pelupessy et al. 2007).

The lifetime of this new DM powered phase of stellar evolution, prior to the onset of fusion, is $\sim 10^{6}$ years. The stars are very bright, $\sim 10^{6} L_{\odot}$, and relatively cool, (600010,000)K (as opposed to standard Pop III stars whose surface temperatures exceed $30,000 K)$. Reionization during this period is likely to be slowed down, as these stars can heat the surroundings but not ionize them. One can thus hope to find DS and differentiate them from standard Pop III stars; perhaps some even still exist to low redshifts.

\section{Later stages: Capture}

There is another possible source of DM in the first stars: capture of DM particles from the ambient medium. Whereas capture is negligible during the pre-mainsequence phase, 
once fusion sets in it can be important, depending on the value of the scattering cross section of DM with the gas. Two simultaneous papers (Freese, Spolyar, \& Aguirre 2008, Iocco 2008) found the same basic idea: the DM luminosity from captured WIMPs can be larger than fusion for the DS. Two uncertainties exist here: the scattering cross section, and the amount of DM in the ambient medium to capture from $\dagger$. DS studies including capture have assumed the maximal scattering cross sections allowed by experimental bounds and ambient DM densities that are never depleted. With these assumptions, DS evolution models with DM heating after the onset of fusion have now been studied in several papers (Iocco et al. 2008, Taoso et al. 2008, Yoon et al. 2008) which were posted during the conference and will be discussed in the talk by Fabio Iocco. We have been pursuing similar research with Alex Heger on DS evolution after the onset of fusion.

\section{Conclusion}

The line of research we began in Florence almost two years ago is reaching a very fruitful stage of development. Dark matter can play a crucial role in the first stars. The first stars to form in the universe may be Dark Stars: powered by DM heating rather than by fusion. Our work indicates that they may be very large $\left(850 M_{\odot}\right.$ for $100 \mathrm{GeV}$ mass WIMPs). The connections between particle physics and astrophysics are ever growing!

\section{References}

Spolyar, D., Freese, K., \& Gondolo, P. astro-ph/0705.0521, 2008, Phys. Rev. Lett., 100, 051101

K. Freese, P. Gondolo, \& Spolyar, D. 2008, AIP Conf.Proc., 990, 42

Ripamonti, E. \& Abel, T. astro-ph/0507130.

Barkana, R. \& Loeb, A. 2001, Phys. Rep., 349, 125

Bromm, V. \& Larson, R. B. 2004, ARAA, 42, 79

Yoshida, N., Omukai, K., Hernquist, L., \& Abel, T. 2006, ApJ, 652, 6

Navarro, J. F., Frenk, C. S., \& White, S. D. M. 1996, ApJ, 462, 563

Blumenthal, G. R., Faber, S. M., Flores, R., \& Primack, J. R. 1986, ApJ, 301, 27

Abel, T., Bryan, G. L., \& Norman, M. L. 2002, Science, 295, 93

Gao, L., Abel, T., Frenk, C. S., Jenkins, A., Springel, V., \& Yoshida, N. 2007, MNRAS. 378, 449

Natarajan, A., Tan, J., \& O'Shea, B. 2008, arXiv:0807.3769 [astro-ph]

Freese, K., Gondolo, P., Sellwood, J., \& Spolyar, D. 2008, arxiv:0805.3540 [astro-ph]

Ripamonti, E., Mapelli, M., \& Ferrara, A. 2007, MNRAS, 375, 1399

Hollenbach, D. \& McKee, C. F. 1979, ApJ Suppl., 41, 555

Yoshida, N., Freese, K., Gondolo, P., \& Spolyar, D. 2008, in preparation

Freese, K., Bodenheimer, P., Spolyar, D., \& Gondolo, P. 2008, arxiv:0806.0617 [astro-ph]

Heger, A. \& Woosley, A. 2002, ApJ, 567, 532

Li, Y. X. et al. 2007, ApJ, 665, 187

Pelupessy, F. I., Matteo, T. Di, \& Ciardi, B. 2007, arXiv:astro-ph/0703773.

Freese, K., Spolyar, D., \& Aguirre, A. 2008, arXiv:0802.1724 [astro-ph]

Iocco, F. 2008, ApJ Letters, 677, 1

Iocco, F., Bressan, A., Ripamonti, E., Schneider, R., Ferrara, A., \& Marigo, P. 2008, arXiv:0805.4016

Taoso, M., Bertone, G., Meynet, G., \& Ekstrom, S. 2008, arXiv:0806.2681 [astro-ph]

Yoon, S., Iocco, F., \& Akiyama, S. 2008, arxiv:0806.2662 [astro-ph]

$\dagger$ Unlike the annihilation cross section, which is set by the relic density, scattering is to some extent a free parameter set only by bounds from direct detection experiments. 